\title{
EUS FNA of Altered Left Adrenal Gland Morphology Suggests Amending CT and PET-CT Attenuation Threshold Values That Predict Malignancy
}

\author{
Ferga C. Gleeson ${ }^{1 *}$, Allison J. Clapp ${ }^{2}$, Robert C. Murphy ${ }^{2}$, Jonathan E. Clain ${ }^{1}$, Prasad G. Iyer ${ }^{1}$, \\ Elizabeth Rajan ${ }^{1}$, Mark D. Topazian ${ }^{1}$, Kenneth K. Wang ${ }^{1}$, Michael J. Levy ${ }^{1}$ \\ ${ }^{1}$ Division of Gastroenterology and Hepatology, Mayo Clinic College of Medicine, Rochester, USA; ${ }^{2}$ Department of Radiology, \\ Mayo Clinic College of Medicine, Rochester, USA. \\ Email: "gleeson.ferga@mayo.edu
}

Received September $12^{\text {th }}, 2012$; revised October $14^{\text {th }}, 2012$; accepted October $23^{\text {rd }}, 2012$

\begin{abstract}
Introduction: In the setting of an extra-adrenal malignancy, it is a recognized clinical challenge to try and distinguish a benign adrenal mass from a metastatic deposit. Current non-invasive diagnostic tools for adrenal gland evaluation include CT, MRI, PET and PET-CT. Diagnostic interpretative error can occur as evaluations rarely have complete cytologic or histologic correlation for concordance purposes. Aims: To establish the performance characteristics of noncontrast CT attenuation values (Hounsfield units-HU) and the optimal PET-CT maximum standard uptake value $\left(\mathrm{SUV}_{\max }\right)$ for predicting adrenal malignancy when correlated with adrenal gland endoscopic ultrasound fine needle aspiration (EUS FNA) cytology results. Methods: A prospectively maintained EUS database was reviewed to identify consecutive patients who underwent a left adrenal gland FNA. Non-contrast CT attenuation values and $\mathrm{SUV}_{\max }$ scores were calculated. EUS FNA cytology results were used as the reference standard for determining the presence of benign versus malignant adrenal gland status. Results: Sixty-two patients ( $69 \pm 11$ years) underwent adrenal EUS FNA, 34 $(54.8 \%)$ of whom had a clinically suspected or established extra-adrenal malignancy. Non-invasive imaging was suggestive of abnormal adrenal morphology or altered PET-CT FDG activity in 45 (72.6\%) patients. Elevated attenuation values $(\geq 10 \mathrm{HU})$ by non-enhanced CT had a sensitivity and specificity of $100 \%$ and $34.6 \%$, respectively. The $\mathrm{SUV}_{\max }$ for malignant altered morphology was significantly higher than that for benign lesions $[8.5 \pm 3.1 \mathrm{vs} 3.3 \pm 0.7$; $(\mathrm{p}=$ 0.0001 )]. ROC curve analysis indicated that an optimum cutoff $\mathrm{SUV}_{\max }$ of $\geq 4.1$ (AUC 0.92) yielded the best power distinction for malignancy with a sensitivity and specificity of $89 \%$ and $100 \%$. Conclusion: When evaluating altered adrenal morphology by non-invasive methods, the performance characteristics of elevated CT attenuation values are suboptimal. But by adopting a $\mathrm{SUV}_{\max }$ cut-off value of $\geq 4.1$ could potentially improve such characteristics to detect malignancy.
\end{abstract}

Keywords: Altered Adrenal Morphology; Endoscopic Ultrasound Fine Needle Aspiration; Unenhanced CT Attenuation Value; PET Standardized Uptake Value

\section{Introduction}

Incidentally identified adrenal masses noted by non-invasive imaging or by autopsy are found in $0.5 \%-10 \%$ of the population [1-3]. The dilemma therefore, is in the setting of an established or questionable extra-adrenal malignancy to differentiate between a benign adrenal mass or metastatic deposit. Characterization of altered adrenal morphology is clearly important for disease management and prognosis. Current non-invasive imaging modalities include computed tomography (CT), magnetic resonance imaging (MRI), F-18 fluorodeoxyglu-

"Corresponding author. cose positron emission tomography (F-18 FDG PET) and more recently integrated PET-CT.

Unenhanced CT and MRI images have been used to distinguish lipid containing adrenal adenomas from metastases and other lesions on the basis of the presence of fat [4]. An unenhanced CT attenuation threshold of $\leq 10$ Hounsfield Units (HU) is used as a discriminating index to diagnose a lipid rich adrenal adenoma [3,5-7]. Outof-phase MRI imaging shows signal dropout due to the presence of intracellular lipid in lipid rich adenomas. Unfortunately, the performance characteristics are relatively poor, as $30 \%$ of adenomas are lipid poor, and in addition to malignant pathology, have a higher attenua- 
tion $(\mathrm{HU})$ value $[8,9]$. Lesion size and shape may be helpful in differentiating a benign from a malignant adrenal mass, but again they are not specific [10].

The limited accuracy of lesion localization using PET alone, due to the lack of precise anatomical landmarks led to the development of integrated PET-CT to provide anatomic-metabolic information which is considered to be more sensitive and specific than PET alone, when depicting adrenal gland metastasis [11]. However, false positive interpretation even with PET-CT can occur as studies rarely have complete cytological or histological correlation for concordance purposes [11-13]. Such examples include; $5 \%$ of adrenal adenomas, adrenal endothelial cysts, adrenal cortical hyperplasia without chronic inflammatory cell infiltration, phaeochromocytoma and inflammatory or infectious lesions [14]. PET-CT with additional maximum standard uptake value analysis (SUVmax score) is thought to potentially improve the diagnostic accuracy of adrenal lesions in patients with cancer (Table 1) [15-21]. In spite of this, there is no universally accepted $\mathrm{SUV}_{\max }$ score to predict malignancy, at any site or a specific score dedicated for the evaluation of the adrenal gland [18]. However, by convention, a common threshold $\mathrm{SUV}_{\max }$ score $>2.5$ is used to distinguish a benign from a malignant process [22-26].

The left adrenal gland may be sampled using CT or trans abdominal ultrasound guided fine needle aspiration (FNA), by open or laparoscopic adrenalectomy and more recently endoscopic ultrasound (EUS) guided FNA [10, 27-35]. EUS FNA is accepted as a highly specific and safe technique for confirming the diagnosis of metastatic disease to the adrenal glands [32]. While lesion size ( $\geq 30$ $\mathrm{mm}$ ) and EUS echodensity (hypoechoic) poorly distinguish malignant from benign masses, altered adrenal gland morphology (loss of classic seagull configuration) is highly predictive [10]. EUS FNA of an enlarged left adrenal gland in patients with established or suspected lung cancer was shown to alter the TNM stage and treatment in $70 \%$ and $48 \%$ of patients, respectively [28]. EUS guided ethanol ablation of left adrenal metastasis from non-small cell lung carcinoma has also been reported [36].

The aim of the study was to establish the sensitivity and specificity of unenhanced $\mathrm{CT}$ attenuation values and to determine the optimal PET-CT SUV $\mathrm{max}_{\text {score }}$ sor predicting left adrenal gland malignancy as compared to a gold standard consisting of EUS FNA cytology with clinical follow-up.

\section{Patients and Methods}

\subsection{Study Design and Setting}

A prospectively maintained EUS database was reviewed to identify consecutive patients who underwent left adrenal gland EUS FNA from May 1, 2005 to November 1, 2011. EUS examinations were performed by one of eight experienced endosonographers. Radiologic calculation of unenhanced CT attenuation values and FDG PET-CT $\mathrm{SUV}_{\max }$ scores were performed by one radiologist who was blinded to any clinical or pathologic data specifically for the purpose of the study. Clinical demographics, radiologic, EUS and cytologic data, in addition to clinical follow up were retrieved and analyzed.

\subsection{CT Attenuation Analysis of the Left Adrenal Gland}

Utilizing transaxial CT slices from an unenhanced CT, a circular or oval manually defined region of interest was placed in the center of the adrenal mass to obtain $\mathrm{CT}$ attenuation values in Hounsfield units (HU). A CT slice was selected that allowed at least two thirds of the adrenal mass to be evaluated. An attenuation value of $\geq 10$ HU was considered to represent a non-adenomatous lesion.

\subsection{Quantitative Analysis of F18-FDG PET Based on Standardized Uptake Values (SUVs)}

As per standard technique based on a qualitative visual

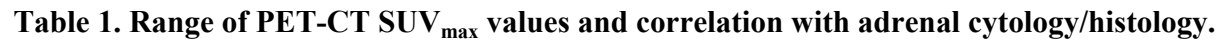

\begin{tabular}{|c|c|c|c|c|c|c|}
\hline Author & $\mathrm{n}$ & $\mathrm{SUV}_{\text {max }}$ & $\begin{array}{l}\text { Cytology or pathology } \\
\text { correlation } n, \%\end{array}$ & Sensitivity $\%$ & Specificity \% & Follow Up Duration \\
\hline Perrii $^{2011}$ & 93 & $>2.8$ & $10(11 \%)$ & 100 & 72 & 6 months \\
\hline Nguyen $^{2011}$ & 36 & $>2.2$ & $5(14 \%)$ & 100 & 93 & $2-166$ weeks \\
\hline Ansquer ${ }^{2010}$ & 81 & $>3.3$ & $49(61 \%)$ & 93 & 78 & 9 months \\
\hline Brady $^{2009}$ & 147 & $>3.1$ & $12(8 \%)$ & 97 & 76 & 1 year \\
\hline Okada $^{2009}$ & 30 & $>2.5$ & 0 & 89 & 94 & 1 year \\
\hline Jana $^{2006}$ & 92 & $>3.4$ & $13(14 \%)$ & 95 & 86 & 1 year \\
\hline
\end{tabular}


assessment, PET findings were interpreted as positive if the FDG uptake in an adrenal lesion was greater than or equal to that in the generalized liver and as negative, if lesion uptake was less than that of the liver. A PET-CT $\mathrm{SUV}_{\max }$ score was calculated by creating an elliptic region of interest over a minimum of two thirds of the adrenal lesion.

\subsection{Diagnostic Gold Standard-EUS FNA Cytology}

An electronic multi-element curved-linear US echoendoscope (GF-UC140P-AL5 or GF-UC 160P-AT8; Olympus) was used to perform FNA with an 8-cm-long, 22gauge needle (Echotip; Cook Endoscopy, Winston-Salem, NC). Altered adrenal morphology was considered, if the standard seagull configuration was lost to include 1) a general enlargement of the gland akin to a mass; 2) presence of a focal nodule or 3) presence of limb thickening. EUS FNA cytology results were used as the reference standard for final classification of the altered adrenal morphology when determining the presence of benign versus malignant adrenal gland status. Cytology specimens were interpreted as either positive for malignnancy, suspicious for malignancy, atypical, negative for malignancy, or inadequate cellularity for interpretation. For statistical purposes, results that were positive or suspicious for malignancy were considered to be positive and the remaining atypical, negative for malignancy, or inadequate cellularity for interpretation cases were considered to be negative for malignancy.

\subsection{Statistical Analysis}

Continuous variables were reported as mean (SD) or median (interquartile range) and compared by using the Student $t$ test or Mann Whitney $U$ test. Categorical variables were reported as frequency (\%) and were compared by either a 2-tailed Fisher exact test or Pearson $\chi^{2}$ test, when appropriate. An ROC curve analysis was performed to evaluate $\mathrm{HU}$ values and $\mathrm{SUV}_{\max }$ score data to yield the best power distinction for malignant cytology. All tests were 2-sided, with $P=0.05$ as the criterion standard for determining significance. The statistical software package JMP Version 9 (SAS Institute Inc., Cary, NC) and MedCalc were used for statistical analysis.

\section{Results}

Sixty-two consecutive patients $(69 \pm 10.5$ years $)$ underwent left adrenal EUS FNA to evaluate a mass $(n=31)$, nodule $(n=17)$ or limb thickening $(n=14)$. Prior to the EUS examination, $34(54.8 \%)$ patients had a clinically suspected or established extra-adrenal malignancy including lung $(\mathrm{n}=21 ; 55 \%)]$, esophageal $(\mathrm{n}=7)$, gastric $(n=2)$ and one patient each for gastric gastrointestinal stromal tumor (GIST), pancreas neuroendocrine tumor (NET), breast cancer and colon cancer. Fifty-seven (92\%) patients had non-invasive imaging with CT $[\mathrm{n}=35$, $(12 / 35$ performed with IV contrast)] or PET-CT $(n=22)$ within 4 weeks prior to EUS FNA.

With a median of 3 (IQR 2 - 5) FNA passes, in the absence of reported complications, the cytological findings were negative for malignancy $(n=41)$, positive or suspicious for malignancy $(\mathrm{n}=15)$, atypical $(\mathrm{n}=2)$ or inadequate cellular yield $(n=4)$. The positive for malignancy cases included metastases from non small cell lung carcinoma $(n=10)$, esophageal adenocarcinoma $(n=2)$, pancreas adenocarcinoma $(n=1)$, breast ductal carcinoma $(n=1)$ and a GIST $(n=1)$. Positive cytology was seen more frequently in patients with a personal history or a newly suspected or confirmed cancer diagnosis $(13 / 15$ vs $21 / 47, p=0.006)$.

\subsection{Evaluation of Unenhanced CT Findings Pre EUS}

Non-invasive imaging revealed abnormal adrenal morphology or altered F18-FDG activity in 45 patients [(72.6\%) (23/35 CT \& 22/22 PET-CT)] (Figures 1 and 2). The 12 unremarkable CT scans by final report were all performed with IV contrast. The sensitivity and specificity of elevated attenuation values ( $\geq 10 \mathrm{HU}$ ) by non-enhanced CT alone, by non-enhanced CT as part of integrated PET-CT or a combination of non-enhanced CT

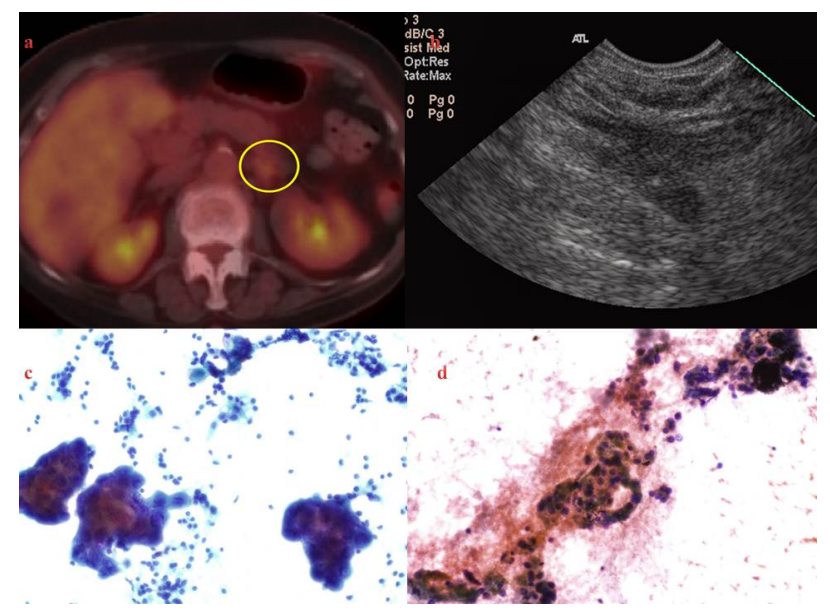

Figure 1. 78-year-old female with primary lung adenocarcinoma and slightly avid uptake of left adrenal gland by PET-CT (a). Review of attenuation values revealed a CT $\mathrm{HU}=13$ and PET $S U V_{\text {max }}=3.2$. EUS revealed a slightly thickened gland (b), positive sub-carinal lymph node for malignancy (c) and a negative left adrenal gland for malignancy. 


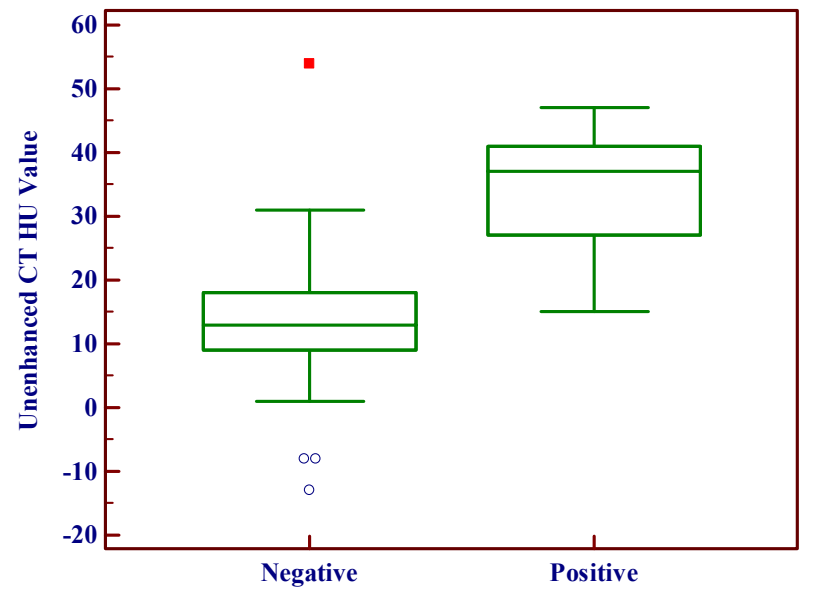

(a)

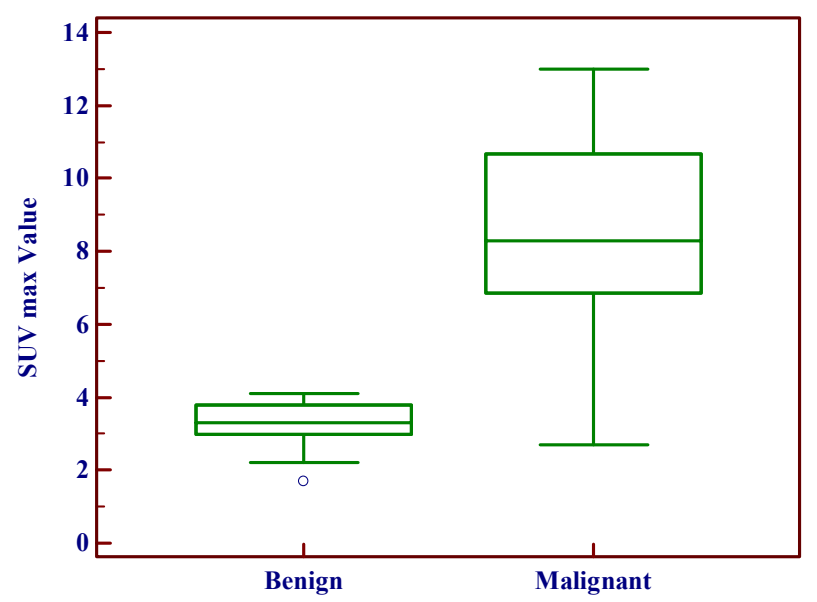

(b)

Figure 2. Unenhanced CT HU and PET SUV $\max$ values with corresponding cytology interpretation.

cases in addition to the non-enhanced CT component from the PET evaluation are documented in Table 2 \& Figure 3. Using FNA cytology results as the gold standard, ROC curve analysis indicated an optimum cutoff HU value $\geq 25$ with an AUC of 0.90 (95\% CI, $0.76-0.98)$ yielded the best power distinction for malignancy to provide a sensitivity and specificity of $90 \%$ and $84.6 \%$, respectively (Figure 4).

\subsection{Quantitative Analysis of PET SUV max $_{\text {ax }}$}

The $\mathrm{SUV}_{\max }$ for malignant altered morphology was $8.5 \pm$ 3.1 , significantly higher $(p=0.0001)$ than that for benign lesions $(3.3 \pm 0.7)$. ROC curve analysis indicated an optimum cutoff $\mathrm{SUV}_{\max } \geq 4.1$ with an AUC of $0.92(95 \%$ CI, 0.72 - 0.99) yielding the best power distinction for malignancy, that provided a sensitivity and specificity of $89 \%$ and 100\%, respectively (Figure 5). If that threshold is adopted the false negative rate would correspond to $1 / 9$ (11\%). However, if a threshold of $\geq 2.5$ was used instead,
Table 2. Sensitivity and specificity of unenhanced CT attenuation values $\geq 10$.

\begin{tabular}{lcc}
\hline & Sensitivity $\%$ & Specificity $\%$ \\
\hline$\geq 10(\mathrm{n}=9 / 14 \%-64 \%)^{1}$ & 100 & 38.5 \\
$\geq 10(\mathrm{n}=18 / 22 \%-82 \%)^{2}$ & 100 & 30.8 \\
$\geq 10(\mathrm{n}=27 / 36 \%-75 \%)^{3}$ & 100 & 34.6 \\
\hline
\end{tabular}

${ }^{1}$ Non-enhanced CT; ${ }^{2}$ Non-enhanced CT when part of integrated PET-CT ${ }^{3}$ Combination of non-enhanced CT \& the non-enhanced CT component from PET-CT evaluation.

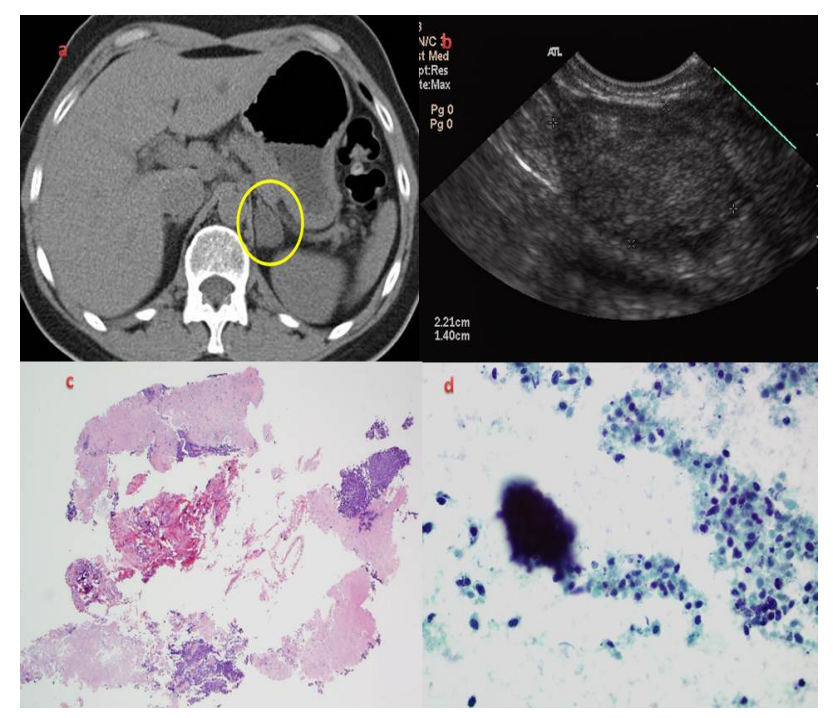

Figure 3. EUS referral: 55 year old female with very bulky subcarinal lymph nodes $(3 \times 2.5 \mathrm{~cm})$ and a (a) left adrenal mass $(2.3 \times 1.6 \mathrm{~cm})$. EUS FNA of the adrenal mass $(\mathrm{b})$ and sub-carinal lymph nodes revealed histologic (subcarinal LN TCB to exclude lymphoma) (c) and cytologic (d) findings diagnostic of an extensively necrotic, metastatic grade 4/4 undifferentiated small cell carcinoma of lung origin. Review of the left adrenal gland $C T$ attenuation value revealed a HU of 15.

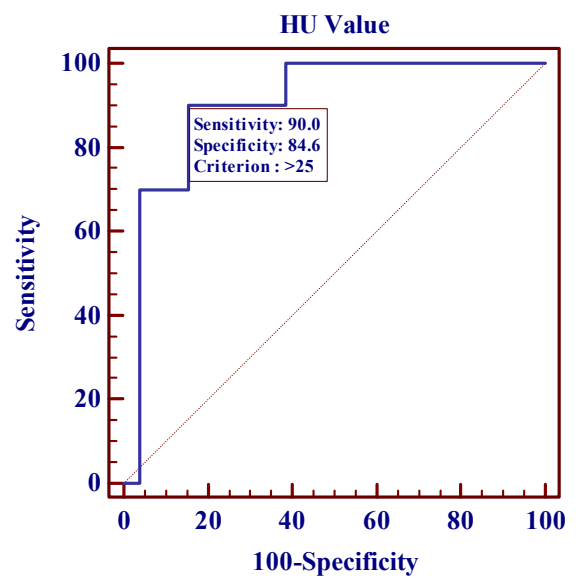

Figure 4. ROC curve analysis of CT HU values. 


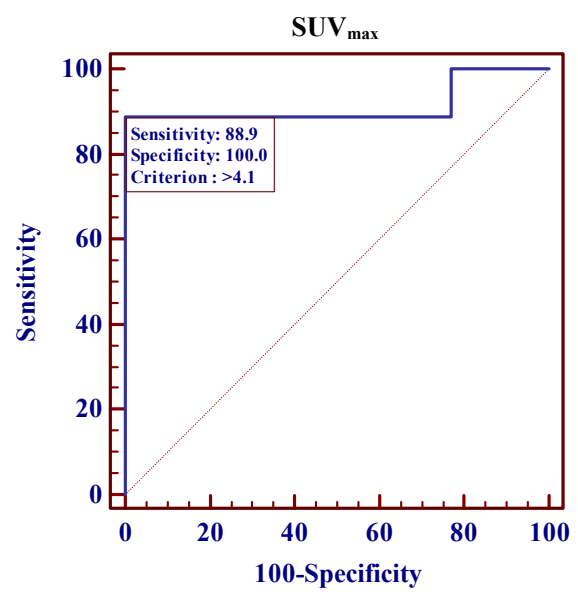

Figure 5. ROC curve analysis of PET SUV $\mathrm{max}_{\operatorname{mal}}$ valu.

as per ROC analysis, then the sensitivity and specificity values correspond to $100 \%$ and $23 \%$, respectively.

\subsection{Unenhanced CT Attenuation plus $S U V_{\text {max }}$ Values for Integrated PET-CT}

Elevated attenuation values ( $\geq 10 \mathrm{HU})$ accompanied by a $\mathrm{SUV}_{\text {max }}$ score $\geq 4.1$ were noted in $8 / 9(88.9 \%)$ patients with malignant cytology, revealing a sensitivity and specificity of $89 \%$ and $85 \%$, respectively. Elevated attenuation values ( $\geq 25 \mathrm{HU}$ ) accompanied by a $\mathrm{SUV}_{\max }$ score $\geq 4.1$ were again noted in $8 / 9(88.9 \%)$ patients with malignant cytology, revealing a sensitivity and specificity of $88.9 \%$ and $92.3 \%$, respectively (Figure 6).

\subsection{Unsuspected Altered Adrenal Morphology at EUS}

Seventeen of 62 patients had unsuspected altered adrenal morphology at EUS, 4 of whom had evidence of metastatic disease, from either non-small cell lung cancer $(\mathrm{n}=$ $2)$, breast ductal carcinoma $(\mathrm{n}=1)$ or a GIST $(\mathrm{n}=1)$, thus altering management in $4 / 62(6.5 \%)$ patients.

\subsection{Clinical Follow Up of Non-Malignant EUS Cytology}

Patients were followed clinically for $25 \pm 18.5$ months, during which period 9/45 (19\%) patients died [pancreas adenocarcinoma $(n=7)$, cholangiocarcinoma $(n=1)$, non-small cell lung cancer $(n=1)]$. The remaining patients with negative left adrenal gland cytology had a diagnosis of non-small cell lung cancer lung $(n=13)$, esophageal adenocarcinoma $(n=5)$, pancreas adenocarcinoma $(\mathrm{n}=3)$, and one patient each with gastric, colon, gastric GIST, pancreas NET and rectal adenocarcinoma. Finally, no clinical evidence of extra adrenal malignancy has been noted to date in the remaining 12 patients.

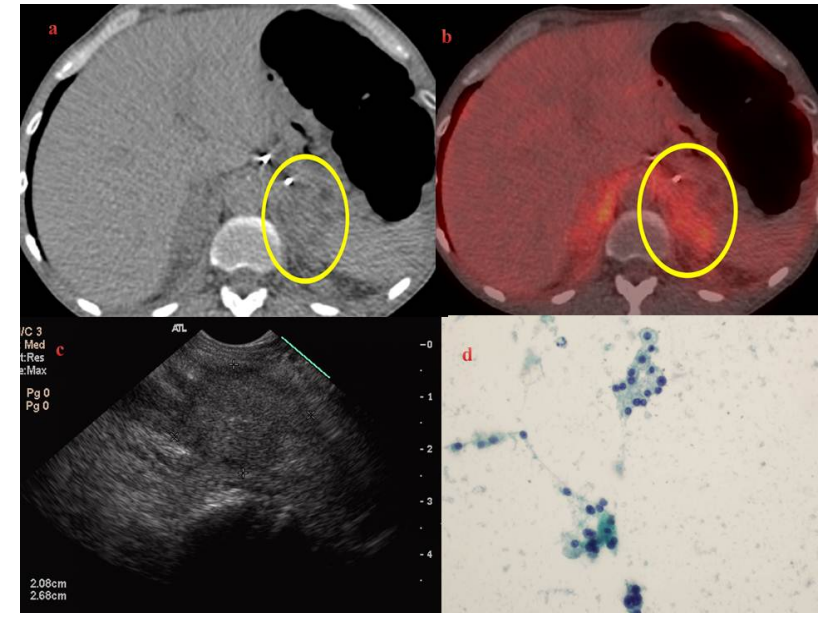

Figure 6. 72-year-old male smoker with a negative CT guided biopsy of a left pleural mass and FDG uptake in several extrapulmonary sites to include bilaterally enlarged adrenal glands (a) \& (b). EUS revealed a $2.7 \mathrm{~cm}$ hypoechoic homogenous left adrenal mass (c) and the right adrenal gland was not visualized. Review of attenuation values revealed a CT HU of 28 and PET SUV $\mathrm{Sax}_{\max }$ of 4.1 with (d) negative left adrenal cytology for malignancy. The final working diagnosis was that of bilateral adrenal hyperplasia, a recognized PET false positive interpretation pitfall, with no evidence of pulmonary malignancy 12 months later.

\section{Discussion}

Altered adrenal morphology in patients with extra adrenal malignancy is a clinical conundrum. Approximately, $50 \%$ of such patients will have metastatic disease to the adrenal gland, but the remainder will have non-metastatic etiologies including cortical adenomas [36,37].

Our study observed that an unenhanced CT attenuation value of $\geq 10 \mathrm{HU}$ for an adrenal lesion is highly sensitive but poorly specific $(31 \%$ - 39\%) for malignancy. The high sensitivity is in accordance with adrenalectomy histopathologic findings when correlated with imaging characteristics [38]. In order to improve specificity, recent studies recommend using cut-off values of $20 \mathrm{HU}$ or $25 \mathrm{HU}$ to achieve specificities of $60 \%$ or $72 \%$, respecttively [9]. Our ROC analysis using cytology as the gold standard indicates that a cutoff value of $25 \mathrm{HU}$ provides a sensitivity and specificity of $90 \%$ and $84.6 \%$, respecttively. In adults, adrenal gland volume and attenuation correlate positively and inversely with age, but the metabolic activity does not alter significantly with age, and therefore PET permits the evaluation of a primary lesion, in addition to metastases as part of non-invasive whole body imaging $[39,40]$.

The presented study revealed an optimum cut off PET $\mathrm{SUV}_{\max }$ of 4.1 (AUC 0.92) as the best power distinction for malignant adrenal lesions (sensitivity 89\%; specificity $100 \%$ ). This value is certainly higher than that re- 
ported for adrenal metastases elsewhere $\left(\mathrm{SUV}_{\max } 2.2\right.$ 3.4 ), but the presented study is the only one with complete cytologic correlation rather than an interval imaging evaluation or clinical follow up. The use of a high $\mathrm{SUV}_{\max }$ threshold may be criticized as it could potentially exclude a considerable number of false-negative results to include hemorrhagic or necrotic metastatic lesions, metastatic lesions $<10 \mathrm{~mm}$ as a critical mass of metabolically active malignant cells is required, and metastases from pulmonary bronchoalveolar carcinoma or carcinoid tumors. Hypothetically, if this high $\mathrm{SUV}_{\max }$ cut off value was the given standard; our false negative rate would correspond to $11 \%$. The ideal threshold $\mathrm{SUV}_{\max }$ to adopt depends on whether one favors a greater sensitivity and corresponding poor specificity or alteratively a greater specificity with associated poor sensitivity. As for any test, this decision is influenced by the manner in which the test results, whether positive or negative, impact the subsequent diagnostic and therapeutic care.

Two studies evaluating PET for mediastinal lung cancer staging using either mediastinoscopy or mediastinal lymphadenectomy as the diagnostic gold standard determined that the highest diagnostic accuracy was achieved with $\mathrm{SUV}_{\max }$ values of 4.5 and 5.3 respectively, rather than the traditional value of $\geq 2.5[41,42]$. When we applied the conventional value of $\geq 2.5$ to the adrenal gland with direct cytological correlation, the specificity value correspond to a poor and clinically unacceptable $23 \%$.

In order to improve PET-CT diagnostic accuracy, Perri and colleagues combined mean SUV values with a CT histogram analysis revealing a sensitivity and sensitivity of $100 \%$ and $97.3 \%$, respectively, leading to a significant reduction in the number of false-positive cases [17]. We evaluated a combination of CT attenuation and $\mathrm{SUV}_{\max }$ values, noting that an attenuation value of $\geq 10 \mathrm{HU}$ or $\geq 25 \mathrm{HU}$ accompanied by a $\mathrm{SUV}_{\max }$ score $\geq 4.1$ revealed a specificity of $89 \%$ and $92 \%$, respectively. Although this was a hypothetical evaluation using values much higher than conventional threshold values, it would still suggest that we should perhaps lower our threshold for sampling the adrenal gland, as it could characterize such lesions with greater confidence. Limitations of our study include: referral bias, the small cohort size, a retrospective evaluation of our EUS and cytological data and the absence of the ultimate gold standard, that of histopathology.

In 1996, Chang and colleagues initially described the EUS characterization and FNA of the adrenal gland [43]. The European Society of Gastrointestinal Endoscopy (ESGE) recently released an EUS clinical guideline supporting sampling the adrenal gland [44]. EUS guided sampling has some advantages over image guided percutaneous biopsy. EUS obviates the need for needle traversal through the kidney or lung. Thus, complications such as perinephric hemorrhage, pneumothorax ( $5 \%$ risk) and hemothorax rarely develop. In addition, EUS does not require a saline injection to expand the posterior paravertebral space to facilitate sampling as used by some radiologists [45]. The advantages of EUS must be weighed against the cost differential and considered in the context of local expertise.

In conclusion, accurate characterization of altered adrenal morphology in patients with established or possible malignancy is challenging with the current limitations of standard non-invasive imaging. The conventional threshold values used for CT and PET to distinguish a benign from a malignant adrenal entity for unenhanced CT and that of PET-CT are suboptimal when compared to adrenal gland cytologic evaluation by EUS. Our preliminary study suggests that increasing the $\mathrm{HU}$ and $\mathrm{SUV}_{\max }$ cutoff values used for differentiation would significantly increase specificity. Further prospective studies evaluating higher or variable threshold values may be of benefit, but ultimately sampling of the adrenal gland, confers the best management option for the patient in the setting of malignancy to guide the decision making process.

\section{Acknowledgements}

Dr. Tom Sebo and Dr. Diva Salomao, Department of Anatomic/Clinical Pathology, Mayo Clinic College of Medicine, Rochester, MN.

\section{REFERENCES}

[1] C. Davenport, A. Liew, B. Doherty, et al., "The Prevalence of Adrenal Incidentaloma in Routine Clinical Practice," Endocrine, Vol. 40, No. 1, 2011, pp. 80-83. doi:10.1007/s12020-011-9445-6

[2] S. Bovio, A. Cataldi, G. Reimondo, et al., "Prevalence of Adrenal Incidentaloma in a Contemporary Computerized Tomography Series," Journal of Endocrinological Investigation, Vol. 29, No. 4, 2006, pp. 298-302.

[3] M. Terzolo, A. Stigliano, I. Chiodini, et al., "Italian Association of Clinical Endocrinologists. AME Position Statement on Adrenal Incidentaloma," European Journal of Endocrinology, Vol. 164, No. 6, 2011, pp. 851-870. doi:10.1530/EJE-10-1147

[4] G. W. Boland, M. A. Blake, P. F. Hahn and W. W. MayoSmith, "Incidental Adrenal Lesions: Principles, Techniques, and Algorithms for Imaging Characterization," Radiology, Vol. 249, No. 3, 2008, pp. 756-775. doi:10.1148/radiol.2493070976

[5] E. M. Remer, N. Obuchowski, J. D. Ellis, T. W. Rice, D. J. Adelstein and M. E. Baker, "Adrenal Mass Evaluation in Patients with Lung Carcinoma: A Cost-Effectiveness Analysis," American Journal of Roentgenology, Vol. 174, 2000, pp. 1033-1039.

[6] F. E. Nwariaku, J. Champine, L. T. Kim, S. Burkey, G. 
O'keefe and W. H. Snyder III, "Radiologic Characterization of Adrenal Masses: The Role of Computed Tomography-Derived Attenuation Values," Surgery, Vol. 130, No. 6, 2001, pp. 1068-1071.

doi:10.1067/msy.2001.119189

[7] A. H. Hamrahian, A. G. Ioachimescu, E. M. Remer, et al., "Clinical Utility of Noncontrast Computed Tomography Attenuation Value (Hounsfield Units) to Differentiate Adrenal Adenomas/Hyperplasias from Nonadenomas: Cleveland Clinic Experience," Journal of Clinical Endocrinology \& Metabolism, Vol. 90, No. 2, 2005, pp. 871877. doi:10.1210/jc.2004-1627

[8] G. W. Boland, M. J. Lee, G. S. Gazelle, E. F. Halpern, M. M. McNicholas and P. R. Mueller, "Characterization of Adrenal Masses Using Unenhanced CT: An Analysis of the CT Literature," American Journal of Roentgenology, Vol. 171, No. 1, 1998, pp. 201-204.

[9] S. H. Park, M. J. Kim, J. H. Kim, J. S. Lim and K. W. Kim, "Differentiation of Adrenal Adenoma and Nonadenoma in Unenhanced CT: New Optimal Threshold Value and the Usefulness of Size Criteria for Differentiation," Korean Journal of Radiology, Vol. 8, No. 4, 2007, pp. 328-335. doi:10.3348/kjr.2007.8.4.328

[10] M. A. Eloubeidi, K. R. Black, A. Tamhane, I. A. Eltoum, A. Bryant and R. J. Cerfolio, "A Large Single-Center Experience of EUS-Guided FNA of the Left and Right Adrenal Glands: Diagnostic Utility and Impact on Patient Management," Gastrointestinal Endoscopy, Vol. 71, No. 4, 2010, pp. 745-753. doi:10.1016/j.gie.2009.10.022

[11] Y. M. Sung, K. S. Lee, B. T. Kim, et al., "(18)F-FDG PET versus (18)F-FDG PET/CT for Adrenal Gland Lesion Characterization: A Comparison of Diagnostic Efficacy in Lung Cancer Patients," Korean Journal of Radiology, Vol. 9, No. 1, 2008, pp. 19-28. doi:10.3348/kjr.2008.9.1.19

[12] U. Metser, E. Miller, H. Lerman, G. Lievshitz, S. Avital and E. Even-Sapir, "18F-FDG PET/CT in the Evaluation of Adrenal Masses," Journal of Nuclear Medicine, Vol. 47, No. 1, 2006, pp. 32-37.

[13] M. Nakajo, M. Nakajo, Y. Kajiya, et al., "A Black Adrenal Adenoma Difficult to be Differentiated from a Malignant Adrenal Tumor by CT, MRI, Scintigraphy and FDG PET/CT Examinations," Annals of Nuclear Medicine, Vol. 25, No. 10, 2011, pp. 812-817. doi:10.1007/s12149-011-0535-6

[14] S. Chong, K. S. Lee, H. Y. Kim, et al., "Integrated PET-CT for the Characterization of Adrenal Gland Lesions in Cancer Patients: Diagnostic Efficacy and Interpretation Pitfalls," Radiographics, Vol. 26, No. 6, 2006, pp. 1811-1824. doi:10.1148/rg.266065057

[15] M. Okada, T. Shimono, Y. Komeya, et al., "Adrenal masses: The Value of Additional FluorodeoxyglucosePositron Emission Tomography/Computed Tomography (FDG-PET/CT) in Differentiating between Benign and Malignant Lesions," Annals of Nuclear Medicine, Vol. 23, No. 4, 2009, pp. 349-354. doi:10.1007/s12149-009-0246-4

[16] B. Xu, J. Gao, L. Cui, et al., "Characterization of Adrenal
Metastatic Cancer Using FDG PET/CT," Neoplasma, Vol. 59, No. 1, 2012, pp. 92-99. doi:10.4149/neo 2012012

[17] M. Perri, P. Erba, D. Volterrani, et al., "Adrenal Masses in Patients with Cancer: PET/CT Characterization with Combined CT Histogram and Standardized Uptake Value PET Analysis," American Journal of Roentgenology, Vol. 197, No. 1, 2011, pp. 209-216. doi:10.2214/AJR.10.5342

[18] N. C. Nguyen, A. Kaushik, M. K. Wolverson and M. M. Osman, "Is There a Common SUV Threshold in Oncological FDG PET/CT, at Least for Some Common Indications? A Retrospective Study," Acta Oncologica, Vol. 50, No. 5, 2011, pp. 670-677. doi:10.3109/0284186X.2010.550933

[19] M. J. Brady, J. Thomas, T. Z. Wong, K. M. Franklin, L. M. Ho and E. K. Paulson, "Adrenal Nodules at FDG PET/CT in Patients Known to Have or Suspected of Having Lung Cancer: A Proposal for an Efficient Diagnostic Algorithm," Radiology, Vol. 250, No. 2, 2009, pp. 523-530. doi:10.1148/radiol.2502080219

[20] S. Jana, T. Zhang, D. M. Milstein, C. R. Isasi and M. D. Blaufox, "FDG-PET and CT Characterization of Adrenal Lesions in Cancer Patients," European Journal of $\mathrm{Nu}$ clear Medicine and Molecular Imaging, Vol. 33, No. 1, 2006, pp. 29-35. doi:10.1007/s00259-005-1915-8

[21] C. Ansquer, S. Scigliano, E. Mirallié, et al., "18F-FDG PET/CT in the Characterization and Surgical Decision Concerning Adrenal Masses: A Prospective Multicentre Evaluation," European Journal of Nuclear Medicine and Molecular Imaging, Vol. 37, No. 9, 2010, pp. 1669-1678. doi:10.1007/s00259-010-1471-8

[22] I. Kayani, N. Avril, J. Bomanji, et al., "Sequential FDG$\mathrm{PET} / \mathrm{CT}$ as a Biomarker of Response to Sunitinib in Metastatic Clear Cell Renal Cancer," Clinical Cancer Research, Vol. 17, No. 18, 2011, pp. 6021-6028. doi:10.1158/1078-0432.CCR-10-3309

[23] M. Okada, T. Shimono, Y. Komeya, et al., "Adrenal Masses: The Value of Additional FluorodeoxyglucosePositron Emission Tomography/Computed Tomography (FDG-PET/CT) in Differentiating between Benign and Malignant Lesions," Annals of Nuclear Medicine, Vol. 23, No. 4, 2009, pp. 349-354. doi:10.1007/s12149-009-0246-4

[24] P. S. Duarte, H. Zhuang, P. Castellucci and A. Alavi, "The Receiver Operating Characteristic Curve for the Standard Uptake Value in a Group of Patients with Bone Marrow Metastasis," Molecular Imaging and Biology, Vol. 4, No. 2, 2002, pp. 157-160. doi:10.1016/S1095-0397(01)00060-7

[25] R. Hong, J. Halama, D. Bova, A. Sethi and B. Emami, "Correlation of PET Standard Uptake Value and CT Window-Level Thresholds for Target Delineation in CTBased Radiation Treatment Planning," International Journal of Radiation Oncology*Biology*Physics, Vol. 67, No. 3, 2007, pp. 720-726.

[26] D. Wang, C. J. Schultz, P. A. Jursinic, et al., "Initial Experience of FDG-PET/CT Guided IMRT of Head-andNeck Carcinoma," International Journal of Radiation Oncology*Biology*Physics, Vol. 65, No. 1, 2006, pp. 
143-151.

[27] T. L. Ang, T. S. Chua, K. M. Fock, A. K. Tee, E. K. Teo and K. Mancer, "EUS-FNA of the Left Adrenal Gland Is Safe and Useful," Annals Academy of Medicine Singapore, Vol. 36, No. 11, 2007, pp. 954-957.

[28] U. Bodtger, P. Vilmann, P. Clementsen, E. Galvis, K. Bach and B. G. Skov, "Clinical Impact of Endoscopic Ultrasound-Fine Needle Aspiration of Left Adrenal Masses in Established or Suspected Lung Cancer," Journal of Thoracic Oncology, Vol. 4, No. 12, 2009, pp. 1485-1489. doi:10.1097/JTO.0b013e3181b9e848

[29] J. DeWitt, M. Alsatie, J. LeBlanc, L. McHenry and S. Sherman, "Endoscopic Ultrasound-Guided Fine-Needle Aspiration of Left Adrenal Gland Masses," Endoscopy, Vol. 39, No. 1, 2007, pp. 65-71. doi:10.1055/s-2006-945042

[30] M. A. Eloubeidi, S. Seewald, A. Tamhane, et al., "EUSGuided FNA of the Left Adrenal Gland in Patients with Thoracic or GI Malignancies," Gastrointestinal Endoscopy, Vol. 59, No. 6, 2004, pp. 627-633. doi:10.1016/S0016-5107(04)00296-2

[31] M. A. Eloubeidi, L. P. Luz, D. R. Crowe, C. Snowden, D. E. Morgan and P. J. Arnoletti, "Bilateral Adrenal Gland Enlargement Secondary to Histoplasmosis Mimicking Adrenal Metastases: Diagnosis with EUS-Guided FNA," Diagnostic Cytopathology, Vol. 38, No. 5, 2010, pp. 357359.

[32] N. C. Jhala, D. Jhala, M. A. Eloubeidi, et al., "Endoscopic Ultrasound-Guided Fine-Needle Aspiration Biopsy of the Adrenal Glands: Analysis of 24 Patients," Cancer, Vol. 102, No. 5, 2004, pp. 308-314. doi:10.1002/cncr.20498

[33] O. C. Schuurbiers, K. G. Tournoy, H. J. Schoppers, et al., "EUS-FNA for the Detection of Left Adrenal Metastasis in Patients with Lung Cancer," Lung Cancer, Vol. 73, No. 3, 2011, pp. 310-315. doi:10.1016/j.lungcan.2010.12.019

[34] H. Gerke, R. A. Robinson and P. Luo, "Diagnosis of Focal Metastasis to the Adrenal Gland by EUS-Guided Core Biopsy," Gastrointestinal Endoscopy, Vol. 62, No. 3, 2005, pp. 469-471. doi:10.1016/S0016-5107(05)01578-6

[35] E. B. Stelow, S. M. Debol, M. W. Stanley, S. Mallery, R. Lai and R. H. Bardales, "Sampling of the Adrenal Glands by Endoscopic Ultrasound-Guided Fine-Needle Aspiration," Diagnostic Cytopathology, Vol. 33, No. 1, 2005, pp. 26-30. doi: $10.1002 / \mathrm{dc} .20273$

[36] E. L. Artifon, A. M. Lucon, P. Sakai, et al., "EUS-Guided Alcohol Ablation of Left Adrenal Metastasis from NonSmall-Cell Lung Carcinoma," Gastrointestinal Endoscopy, Vol. 66, No. 6, 2007, pp. 1201-1205.

\section{doi:10.1016/j.gie.2007.05.051}

[37] J. T. Lenert, C. C. Barnett Jr., A. P. Kudelka, et al., "Evaluation and Surgical Resection of Adrenal Masses in Patients with a History of Extra-Adrenal Malignancy," Surgery, Vol. 130, No. 6, 2001, pp. 1060-1067. doi: $10.1067 / \mathrm{msy} .2001 .118369$

[38] L. Hammarstedt, A. Muth, H. A. Sigurjónsdóttir, E. Almqvist, B. Wängberg and M. Hellström, "On Behalf of the Adrenal Study Group of Western Sweden. Adrenal Lesions in Patients with Extra-Adrenal MalignancyBenign or Malignant?" Acta Oncologica, Vol. 51, No. 2, 2012, pp. 215-221. doi:10.3109/0284186X.2011.608084

[39] L. Yip, M. E. Tublin, J. A. Falcone, et al., "The Adrenal Mass: Correlation of Histopathology with Imaging," Annals of Surgical Oncology, Vol. 17, No. 3, 2010, pp. 846852. doi:10.1245/s10434-009-0829-2

[40] J. M. Meier, A. Alavi, S. Iruvuri, et al., "Assessment of Age-Related Changes in Abdominal Organ Structure and Function with Computed Tomography and Positron Emission Tomography," Seminars in Nuclear Medicine, Vol. 37, No. 3, 2007, pp. 154-172. doi:10.1053/j.semnuclmed.2007.02.001

[41] D. Hellwig, T. P. Graeter, D. Ukena, et al., "18F-FDG PET for Mediastinal Staging of Lung Cancer: Which SUV Threshold Makes Sense?" Journal of Nuclear Medicine, Vol. 48, No. 11, 2007, pp. 1761-1766. doi:10.2967/jnumed.107.044362

[42] A. S. Bryant, R. J. Cerfolio, K. M. Klemm and B. Ojha, "Maximum Standard Uptake Value of Mediastinal Lymph Nodes on Integrated FDG-PET-CT predicts Pathology in Patients with Non-Small Cell Lung Cancer," Annals of Thoracic Surgery, Vol. 82, 2006, pp. 417-422. doi:10.1016/j.athoracsur.2005.12.047

[43] K. J. Chang, R. A. Erickson and P. Nguyen, "Endoscopic Ultrasound (EUS) and EUS-Guided Fine-Needle Aspiration of the Left Adrenal Gland," Gastrointestinal Endoscopy, Vol. 44, No. 5, 1996, pp. 568-572. doi:10.1016/S0016-5107(96)70010-X

[44] J. M. Dumonceau, M. Polkowski, A. Larghi, et al., "Indications, Results, and Clinical Impact of Endoscopic U1trasound (EUS)-Guided Sampling in Gastroenterology: European Society of Gastrointestinal Endoscopy (ESGE) Clinical Guideline," Endoscopy, Vol. 43, No. 10, 2011, pp. 897-912. doi:10.1055/s-0030-1256754

[45] C. J. Tyng, A. G. Bitencourt, E. B. Martins, P. N. Pinto and R. Chojniak, "Technical Note: CT-Guided Paravertebral Adrenal Biopsy Using Hydrodissection-A Safe and Technically Easy Approach," British Journal of Radiology, 2011. 